\title{
Impact of Cerebral Cardiopulmonary Resuscitation Maneuvers in a General Hospital. Prognostic Factors and Outcomes
}

\author{
Eduardo Bartholomay, Fernando Suparregui Dias, Fábio Alves Torres, Pedro Jacobson, \\ Afonso Mariante, Rodrigo Wainstein, Renato Silva, Luiz Carlos Bodanese \\ Porto Alegre, RS - Brazil
}

\begin{abstract}
Objective - To assess survival of patients undergoing cerebral cardiopulmonary resuscitation maneuvers and to identify prognostic factors for short-term survival.
\end{abstract}

Methods - Prospective study with patients undergoing cardiopulmonary resuscitation maneuvers.

Results - The study included 150 patients. Spontaneous circulation was re-established in $88(58 \%)$ patients, and $42(28 \%)$ were discharged from the hospital. The necessary number of patients treated to save 1 life in 12 months was 3.4. The presence of ventricular fibrillation or tachycardia (VF/VT) as the initial rhythm, shorter times of cardiopulmonary resuscitation maneuvers and cardiopulmonary arrest, and greater values of mean blood pressure (BP) prior to cardiopulmonary arrest were independent variables for re-establishment of spontaneous circulation and hospital discharge. The odds ratios for hospital discharge were as follows: 6.1 (95\% confidence interval [CI] $=2.7$ 13.6), when the initial rhythm was VF/VT; $9.4(95 \%$ CI = 4.1-21.3), when the time of cerebral cardiopulmonary resuscitation was < 15 min; $9.2(95 \%$ CI = 3.9-21.3), when the time of cardiopulmonary arrest was < $20 \mathrm{~min}$; and 5.7 (95\% CI = 2.4-13.7), when BP was > $70 \mathrm{mmHg}$.

Conclusion - The presence of VF/VT as the initial rhythm, shorter times of cerebral cardiopulmonary resuscitation and of cardiopulmonary arrest, and a greater value of BP prior to cardiopulmonary arrest were independent variables of better prognosis.

Keywords: cardiopulmonary arrest, cardiopulmonary resuscitation, in-hospital

Hospital São Lucas da Pontifícia Universidade Católica do Rio Grande do Sul. Mailing address: Eduardo Bartholomay - Rua Desembargador Alves Nogueira, 223/201 - 90470-110 - Porto Alegre, RS - E-mail: ebartholomay@ hotmail.com.br English version by Stela Maris C. e Gandour
Cardiopulmonary arrest has been defined as a sudden lack of spontaneous ventilation and pulse in the great arteries, accompanied by loss of consciousness in one individual at the same time ${ }^{1}$. In many individuals, cardiopulmonary arrest occurs prematurely. Medical science, throughout its history, has accumulated a set of diagnostic and therapeutic measures in an attempt to avoid that tragic end, the so-called cerebral cardiopulmonary resuscitation. Few medical interventions have the potential to save lives like cerebral cardiopulmonary resuscitation does. General survival of patients undergoing cerebral cardiopulmonary resuscitation, defined as hospital discharge after cardiopulmonary arrest, is around $14 \%{ }^{2}$. Five hundred thousand patients have been estimated to undergo cerebral cardiopulmonary resuscitation every year during hospitalization in the United States ${ }^{3}$. The number of patients undergoing out-of-hospital cerebral cardiopulmonary resuscitation maneuvers in the same country is 225000 per year $^{3}$. Therefore, the number of patients undergoing in-hospital cardiopulmonary arrest is double that undergoing out-ofhospital cardiopulmonary arrest. Despite this elevated number, only a few studies analyze the impact of in-hospital cerebral cardiopulmonary resuscitation maneuvers as compared with the innumerable studies published identifying the value of out-of-hospital cerebralcardiopulmonary resuscitation ${ }^{4-6}$. The population undergoing in-hospital cerebral cardiopulmonary resuscitation differs from that undergoing out-of-hospital resuscitation; therefore, to know the characteristics that interfere with the outcomes of in-hospital cerebral cardiopulmonary resuscitation maneuvers is very important. Aiming at assessing the impact of in-hospital cerebral cardiopulmonary resuscitation, at determining survival, the population profile, and etiologies, and at identifying clinical factors implicated in the prognosis of these patients, a prospective study was carried out in a general university-affiliated hospital. 


\section{Methods}

A prospective, observational, descriptive-analytical study was carried out at the Hospital São Lucas of the Pontifícia Universidade Católica do Rio Grande do Sul(PUCRS), which is characterized as a general university-affiliated hospital with 3 units for treatment of critically ill patients: a general intensive care unit with 13 beds, a coronary unit with 15 beds, and a special care unit, incorporated into the emergency department, with 15 beds. The adult admission unit has 533 beds.

The study sample was composed of patients older than 18 years, who had in-hospital cardiopulmonary arrest and underwent cerebral cardiopulmonary resuscitation at the 3 critical care units of the Hospital São Lucas. Patients undergoing cerebral cardiopulmonary resuscitation maneuvers were consecutively included in the sample in the period from September 2000 to August 2001. Those patients, whose unconsciousness was identified out of the hospital, or in whom the cerebral cardiopulmonary resuscitation maneuvers were interrupted early due to the family's wishes or due to the recommendation of the attending physician, were excluded from the study.

The research team was contacted right after the identification of a cardiopulmonary arrest through an internal pager and headed immediately to the site of the arrest. The researchers watched the cardiopulmonary arrests assistance, collecting data referring to the performance of the cerebral cardiopulmonary resuscitation maneuvers prospectively. Information referring to the previous history of the patients was obtained from the physicians responsible for assisting the cardiopulmonary arrest and through the medical records. The patients who succeeded in the cerebral cardiopulmonary resuscitation maneuvers were accompanied daily by the research team until hospital discharge or death. All data obtained were recorded in a standard protocol previously elaborated with 30 objective questions to provide relevant information to the research. When the same patient underwent more than 1 cerebral cardiopulmonary resuscitation maneuver during hospitalization, only the first episode was included as a new case, and the remaining episodes were only followed up aiming at recording the outcomes of death or hospital discharge.

After finishing with data collection, the researchers revised the general mortality rate of the hospital during the period studied through data gathered at the medical records department of the hospital aiming at detecting occasional losses in data collection.

Witnessed cardiopulmonary arrest was defined as the loss of consciousness occurring in the presence of a member of the family or of the hospital staff. The cerebral cardiopulmonary resuscitation maneuvers comprised basic maneuvers (diagnosis of cardiopulmonary arrest, positive pressure ventilation, external chest compressions) and advanced maneuvers (defibrillation, orotracheal intubation, infusion of specific drugs) of cerebral cardiopulmonary resuscitation performed by the medical team aiming at reverting the cardiopulmonary arrest.
The interval estimated for basic resuscitation assistance lasted from identification of unconsciousness to the beginning of ventilation and external chest compression; the interval of advanced assistance lasted from identification of unconsciousness to performance of defibrillation, orotracheal intubation, or drug infusion. The estimated time of cerebral cardiopulmonary resuscitation was established as the period between the beginning of basic aid and the end of cerebral cardiopulmonary resuscitation. The total time of cardiopulmonary arrest was considered the time interval between identification of unconsciousness and the end of cerebral cardiopulmonary resuscitation.

The immediate cause precipitating cardiopulmonary arrest was identified by the physician responsible for the patient, who also consulted the medical records. Those cases in which the cause could not be defined were considered of unknown cause.

Mean blood pressure prior to cardiopulmonary arrest was defined as the last measurement before unconsciousness was determined; mean blood pressure after reversion was that measured 10 minutes after the moment when a supraventricular rhythm with a pulse was established.

The cardiopulmonary arrest was considered in-hospital only when unconsciousness was identified at the hospital.

Success in reverting cardiopulmonary arrest was defined as the maintenance of a supraventricular rhythm or a pacemaker rhythm with a pulse for at least 1 hour after the end of cerebral cardiopulmonary resuscitation ${ }^{7}$. Only the patients being discharged from the hospital were considered survivors of a cardiopulmonary arrest episode.

Prior to hospital discharge, the patients surviving a cardiopulmonary arrest underwent a complete neurological examination to determine the existence of neurological deficits. The following degrees of deficit were considered: degree I- presence of minimum neurological deficit; degree II - patients with a significant neurological deficit, but with preserved self-sufficiency; degree III - patients depending on aid for basic tasks; degree IV - patients in vegetative states and Glasgow coma scale below 8; and degree V patients diagnosed with encephalic death ${ }^{8}$.

The study was submitted to and approved by the Committee on Ethics of the Hospital with no need for patients' written informed consent because of the characteristics of the study. All surviving patients or family members, or both, were informed about their participation in the study, and those who did not want to participate were excluded.

The statistical analysis was performed with SPSS software version 9.0. In the univariate analysis, the Student $t$ test and the chi-square test were used for continuous and dichotomous variables. Logistic regression (Foroard Wald method) for quantitative variables and multiple variance (Manova) for qualitative variables were used for multivariate analysis. The choice of the variables analyzed in the univariate form was based on information obtained in the literature review of similar studies. The variables selected for multivariate analysis were those that reached a $<0.4$ in univariate analysis. A $p<0.05$ was considered significant. 


\section{Results}

The study comprised 150 patients with cardiopulmonary arrest, who underwent in-hospital cerebral cardiopulmonary resuscitation. The sample equally represented the 4 units investigated as follows: 30 (20\%) patients from the intensive care unit, 33 (22\%) from the coronary unit, 44 (29\%) from the special care unit, and $43(28 \%)$ from the admission unit.

Ninety patients (60\%) were males. The mean hospitalization time of these patients prior to cardiopulmonary arrest was $16 \pm 28$ days. The diseases identified as the major causes of cardiopulmonary arrest were as follows: coronary artery disease in $46(30.6 \%)$ patients; septic shock in 40 (26.6\%); pulmonary thromboembolism in $15(10 \%)$; and heart failure in $12(8 \%)$. The following diseases $(21.5 \%)$ were also observed: hyperpotassemia, hypovolemic shock, stroke, pneumothorax, cardiac tamponade, and unknown causes in 5 (3.3\%) patients (fig. 1).

In regard to the cardiac rhythm identified in the initial monitoring of cardiopulmonary arrest we found, in decreasing order: asystolia, ventricular fibrillation or tachycardia, and pulseless electric activity (fig. 2).

The mean time intervals between the detection of unconsciousness and the beginning of the basic and advanced cerebral cardiopulmonary resuscitation maneuvers were, respectively, 1 minute and 2 minutes and 30 seconds. The mean times of cardiopulmonary arrest and cerebral cardiopulmonary resuscitation, and the mean values of blood pressure are shown in table I.

In 88 patients, a supraventricular rhythm was obtained for more than 1 hour, corresponding to an initial success rate of 58\%. Forty-two patients were discharged from the hospital, representing $28 \%$ of the total population and $48 \%$ of the patients were resuscitated with initial success. The mean time of survival of the patients who obtained initial success was $7.5 \pm 14$ days. The mean blood pressure obtained after successful cerebral cardiopulmonary resuscitation maneuvers was $78 \mathrm{mmHg}$. The neurological examination

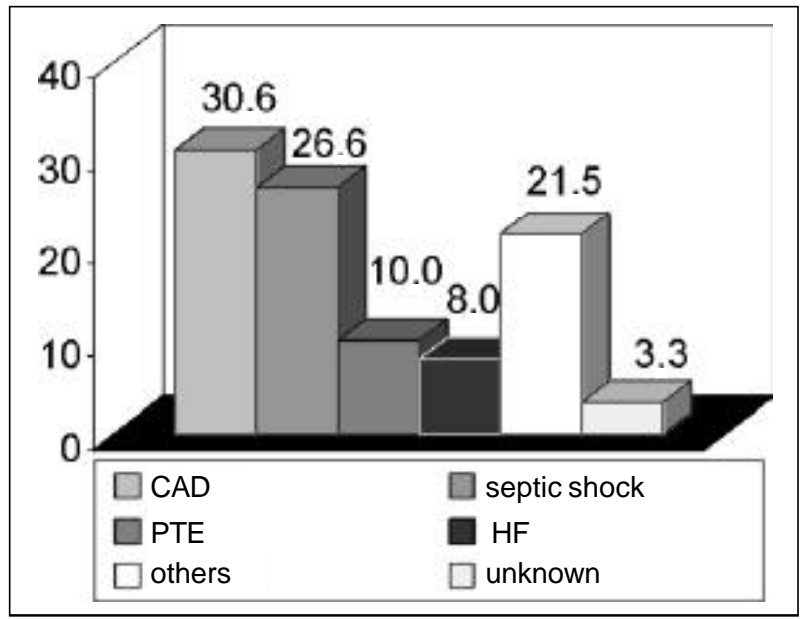

Fig. 1 - Major causes of cardiopulmonary arrest. CAD - coronary artery disease; PTE - pulmonary thromboembolism; $\mathrm{HF}$ - heart failure. performed on the day preceding hospital discharge detected a neurological deficit attributed to the episode of cardiopulmonary arrest in 8 (19\%) patients, who were divided into the following categories: 2 type I patients, 1 type II patient, 4 type III patients, and 1 type IV patient.

Figure 3 depicts in a schematic manner the results of cerebral cardiopulmonary resuscitation maneuvers, including the excluded cases and those lost during the study.

Table II shows the quantitative and qualitative variables observed with multivariate analysis for the outcomes initial success and hospital discharge after in-hospital cerebral cardiopulmonary resuscitation maneuvers.

When the initial rhythm of cardiopulmonary arrest was ventricular fibrillation or tachycardia, the success rate of initial reversion was $87 \%$ ( 35 patients), with a better prognosis in regard to the rhythms of asystolia and pulseless electric activity $(\mathrm{p}=0.001)$. Asystolia had an even greater chance of initial success as compared with pulseless electrical activity ( $\mathrm{p}=0.001$ ). In regard to the outcome hospital discharge, the initial rhythm of ventricular fibrillation or tachycardia had a rate of success of 58\%, also superior to that of other rhythms (pulseless electric activity and asystolia), both with a $16 \%$ rate $(\mathrm{p}=0.001)$.

The shortest time of cerebral cardiopulmonary resuscitation maneuvers and the total time of cardiopulmonary arrests showed statistical significance $(\mathrm{p}=0.001)$ in regard to

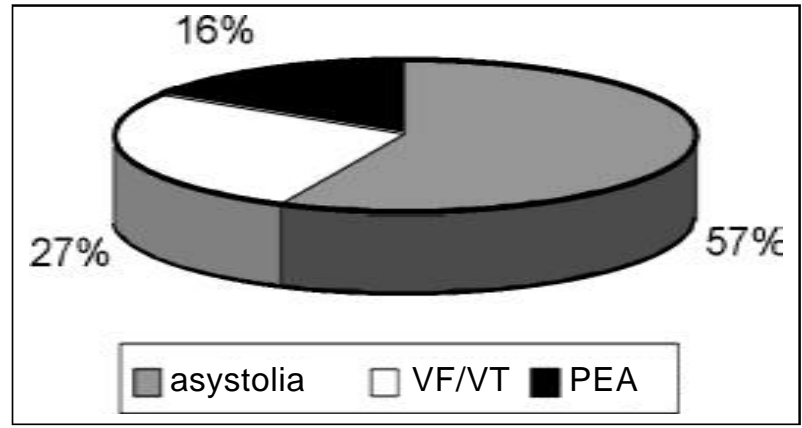

Fig. 2 - Initial cardiac rhythms found in cardiopulmonary arrest. VF/VT - ventricular fibrillation or tachycardia; PEA - pulseless electrical activity.

\begin{tabular}{|lcc|}
\hline \multicolumn{3}{|c|}{$\begin{array}{c}\text { Table I - Characteristics of the cerebral cardiopulmonary } \\
\text { resuscitation maneuvers }\end{array}$} \\
\hline Variable & Mean & Standard deviation \\
\hline Age (years) & 62 & 16 \\
Time of hospitalization prior to CPA (days) & 16 & 28 \\
Interval until basic CCPR (minutes) & 2.4 & 25 \\
Interval until advanced CCPR (minutes) & 4.4 & 1.25 \\
Time of CCPR (minutes) & 25 & 17 \\
Total time of CPA (minutes) & 27 & 19 \\
MBP prior to CPA (mmHg) & 73 & 32 \\
Time elapsed from the last measurement & 60 & 93 \\
of MBP prior to CPA (minutes) & 60 \\
\hline CPA- cardiopulmonary arrest; CCPR- cerebral cardiopulmonary resuscita- \\
tion; interval until basic and advanced CCPR - time interval estimated from \\
identification of unconsciousness to the beginning of basic and advanced \\
maneuvers; MBP- mean blood pressure. \\
\hline
\end{tabular}




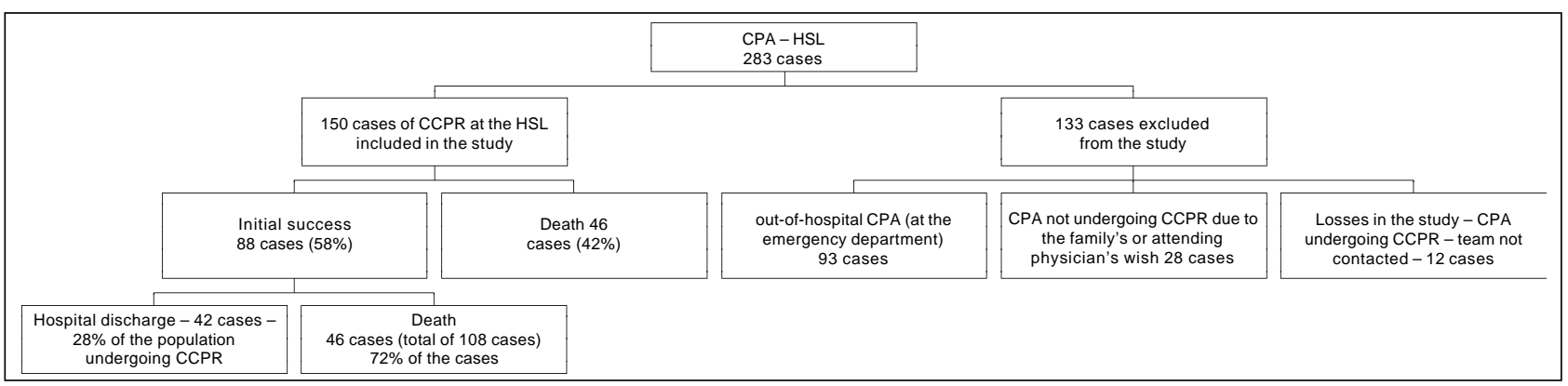

Fig. 3 - General sketch of the outcomes observed after in-hospital cerebral cardiopulmonary resuscitation maneuvers. CPA - cardiopulmonary arrest; CCPR - cerebral cardiopulmonary resuscitation.

the outcomes initial success and hospital discharge. The mean time of cerebral cardiopulmonary resuscitation and total time of cardiopulmonary arrest in initial success and lack of success of the cerebral cardiopulmonary resuscitation maneuvers were, respectively, 16 and 19 minutes and 35 and 38 minutes. For the outcome hospital discharge, the values were 14 and 15 minutes and 28 and 32 minutes. The patients with the greatest values of mean blood pressure prior to cardiopulmonary arrest (mean of $88 \mathrm{mmHg}$ ) were more able to re-establish spontaneous circulation and to be discharged from the hospital as compared with the patients with lower values of mean blood pressure (mean of $65 \mathrm{mmHg}$ ).

To obtain a cut point to calculate the odds ratio of the continuous variables found with significance for the major outcomes $(\mathrm{p}<0.05)$, percentiles were calculated and the cut point values observed were tested, choosing those with a higher statistical significance. The values are shown in tables III and IV with the odds ratio of the qualitative variables.

In regard to the use of antiarrhythmic drugs after cardiopulmonary arrest in patients with ventricular fibrillation or tachycardia as the initial rhythm, maintenance of those drugs was observed in $36 \%$ of the cases, and the rate of hospital discharge was $65 \%$. Amiodarone was used in 22 patients and lidocaine in 4 . When analyzing only the patients with ventricular fibrillation or tachycardia as the

\begin{tabular}{|lcc|}
\hline \multicolumn{2}{|c|}{$\begin{array}{c}\text { Table II - Variables analyzed for the outcomes initial success and } \\
\text { hospital discharge after }\end{array}$} \\
\hline Variables & $\begin{array}{c}\text { Initial success } \\
\text { in CCPR }\end{array}$ & $\begin{array}{c}\text { Hospital discharge } \\
\text { after CCPR }\end{array}$ \\
\hline Age & 0.16 & 0.37 \\
Sex & 0.94 & 0.73 \\
Initial rhythm of CPA & 0.001 & 0.001 \\
Hospitalization time prior to CPA & 0.16 & 0.01 \\
Interval until the beginning of basic & & 0.40 \\
CCPR maneuvers & 0.23 & 0.53 \\
Interval until the beginning of & & 0.001 \\
advanced CCPR maneuvers & 0.60 & 0.001 \\
Time of CCPR & 0.001 & 0.001 \\
Total time of CPA & 0.001 & 0.77 \\
Value of MBP prior to CPA & 0.04 & \\
Value of MBP after CPA & Not assessed & \\
\hline CPA- cardiopulmonary arrest; CCPR- cerebral cardiopulmonary resuscita- & \\
tion; MBP- mean blood pressure. & & \\
\hline
\end{tabular}

initial rhythm, no difference regarding the outcomes initial success and hospital discharge was observed in those in whom the infusion of antiarrhythmic drugs was maintained after cardiopulmonary arrest.
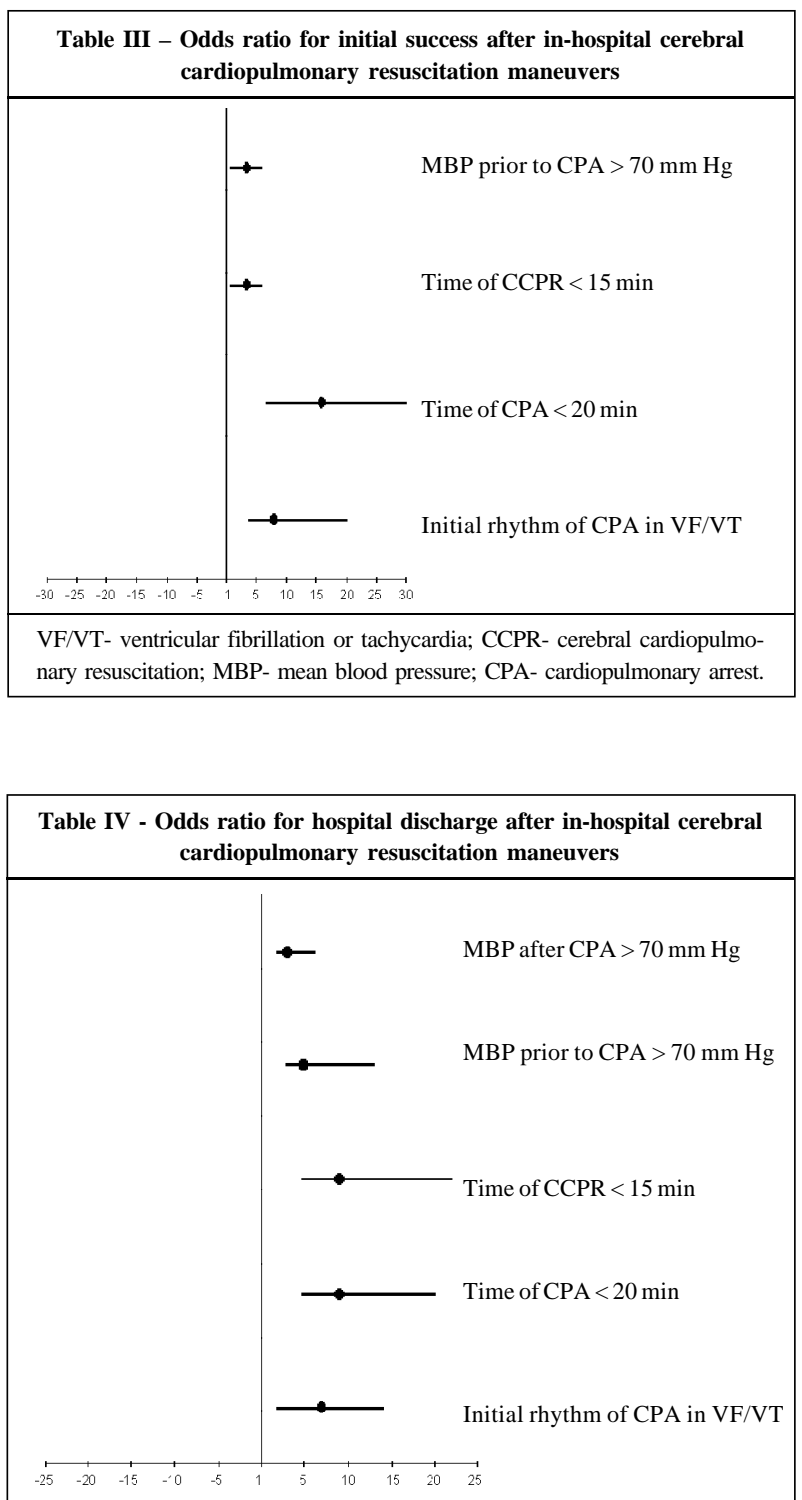

VF/VT- ventricular fibrillation or tachycardia; CCPR- cerebral cardiopulmonary resuscitation; MBP- mean blood pressure; CPA- cardiopulmonary arrest. 


\section{Discussion}

Several studies analyzing in-hospital cardiopulmonary arrest $^{9,10}$ have considered coronary artery disease, sepsis, and heart failure the major causes of cardiopulmonary arrest; however, the presence of pulmonary thromboembolism as one of the most frequently found diseases is not usual. Greater awareness of pulmonary thromboembolism as a cause of cardiopulmonary arrest, especially in the cases of pulseless electric activity, and the appearance of computed angiotomography increased the number of diagnoses of this disease, which might have been previously underestimated. The diagnosis of pulmonary thromboembolism as a cause of cardiopulmonary arrest is of fundamental importance because the possibility of performing catheter thrombectomy or using thrombolytic agents, or both, currently allows a more rapid and effective management. The use of thrombolytic agents in cardiopulmonary arrest was prospectively tested by Böttiger et $\mathrm{al}^{11}$ in 90 patients. Those authors showed the safety of the drug even in prolonged cardiopulmonary arrest, and a possible benefit in the outcome survival in patients with cardiopulmonary arrest due to acute myocardial infarction or pulmonary thromboembolism.

Unlike the results uniformly observed in regard to the initial rhythm found in some studies assessing out-ofhospital cardiopulmonary arrest, in which ventricular fibrillation or tachycardia appears as the initial rhythm in 80-90\% of the cases, the studies analyzing in-hospital cardiopulmonary arrest report different results ${ }^{3,4,6}$. Most patients with out-of-hospital cardiopulmonary arrest have sudden death secondary to ischemic heart disease; however, in the inhospital population, a great diversity of comorbidities exists, which ends up determining the initial rhythm of cardiopulmonary arrest ${ }^{3,5,6}$. Therefore, while in the study by Andreásson et al ${ }^{9} 68 \%$ of the cases have ventricular fibrillation or tachycardia as the initial rhythm, in a Brazilian study ${ }^{12}$ asystolia was the most frequently found rhythm, corresponding to $43 \%$ of the cases. In another prospective sample of 600 patients followed up by Cooper et al ${ }^{7}$, pulseless electric activity was found as the initial rhythm in $48 \%$ of the cases. In our sample, asystolia was the initial rhythm most frequently found (57\%), followed by ventricular fibrillation or tachycardia (27\%), and pulseless electric activity (16\%). The different characteristics of the hospitals, populations, and comorbidities of the patients hospitalized may be responsible for the different findings in regard to the initial rhythm of cardiopulmonary arrest. Initial reversion after cardiopulmonary arrest was obtained in $58 \%$ of the patients, and $28 \%$ of the cases were discharged from the hospital, which was considered the survival rate in our population. Our indices are in accordance with the results of recent studies ${ }^{7,9,13}$. In a meta-analysis of 62 studies assessing the results of in-hospital cerebral cardiopulmonary resuscitation, a great variation in survival (0-30\%) was identified, and the diversity in the populations studied was considered a determinant factor of the different results ${ }^{14}$.
The better results more recently found may be due to the advances in the general assistance to cardiopulmonary arrest expressed, for example, by the presence of programs of qualification of assistance, by the rapid assistance at the hospitals, and by the still expressive number of patients, who had cardiopulmonary arrest in ventricular fibrillation or tachycardia. Improvement in the statistical design of the studies prospectively performed may also be a cause of the increase in the survival rate after cerebral cardiopulmonary resuscitation, because in retrospective analyses many cases of rapidly reverted cardiopulmonary arrest, especially ventricular fibrillation or tachycardia, were not properly recorded.

Better results in rhythms of a worse prognosis, such as asystolia and pulseless electrical activity, may be closely related to promptness in assistance and establishment of reversible causes, a fundamental measure that should always be emphasized in the attempt to improve the prognosis of these patients.

The small number of patients with neurological deficits in our sample is found in the populations undergoing in-hospital cardiopulmonary arrest, and it translates promptness in the initial phase and in performing basic cerebral cardiopulmonary resuscitation, which aims at maintaining cerebral perfusion while re-establishment of a rhythm accompanied by a pulse is sought ${ }^{7,9,13,15}$. In a 2-year followup of 76 patients discharged from the hospital after in-hospital cardiopulmonary arrest, the absence of neurological deficits greater than type I was the most important marker for survival $(\mathrm{p}<0.0008)^{15}$.

Of the variables detected as independent factors of a better prognosis, the initial rhythm of ventricular fibrillation or tachycardia had a better outcome as compared with asystolia and pulseless electrical activity; this result was expected and only confirms that found in the literature ${ }^{7,9,12,13}$. The shorter times of cardiopulmonary arrest and cerebral cardiopulmonary resuscitation as predictors of a better prognosis are justified because cardiopulmonary arrest due to ventricular fibrillation or tachycardia increases its refractoriness to reversion with the increase in the time of cardiopulmonary arrest ${ }^{16}$. The level of adenosine triphosphate, fundamental for myocyte contraction, decreases with the presence of ventricular fibrillation or tachycardia (rhythms that consume a lot of energy), and no reposition occurs due to the lack of appropriate circulation. The shorter time until basic and advanced aid begins, which in other studies appears as a significant prognostic factor, was not important in our sample. We believe that the promptness with which all patients were assisted, with no significant variation in the assisting time, justifies this result. Defibrillation of cardiopulmonary arrest in ventricular fibrillation or tachycardia in a period shorter than 2 minutes was a significant variable in a prospective study carried out at the Hammersmith Hospital in London. In that study, however, a large variation in the times of defibrillation was observed, especially among the patients in the intensive care unit and in the admission unit ${ }^{17}$.

Higher values of mean blood pressure prior to cardio- 
pulmonary arrest as a factor of a better prognosis may be associated with fewer comorbidities and less hemodynamic instability, which are factors associated with greater mortality after cerebral cardiopulmonary resuscitation ${ }^{11,13}$.

The use of antiarrhythmic drugs as a predictor of longer survival correlates with the presence of more patients with ventricular fibrillation or tachycardia as the initial rhythm, favoring its prognosis. When analyzing only the patients with ventricular fibrillation or tachycardia, that variable was not significant. The number of patients taking amiodarone (22 patients) as compared with those taking lidocaine (4 patients) as an antiarrhythmic drug of maintenance after reversing cardiopulmonary arrest due to ventricular fibrillation or tachycardia is noteworthy. Amiodarone has been established as effective during cardiopulmonary arrest in the ARREST study ${ }^{18}$ and incorporated into the guidelines of the American Heart Association (AHA) since August $2000{ }^{19}$. However, no study so far has supported it as a maintenance antiarrhythmic agent; there remains doubt as to its effectiveness in evidence-based medicine. Lidocaine, an antiarrhythmic agent previously used as the first choice in that situation, also needs important studies establishing its efficacy; this is perhaps the reason why amiodarone can be established as the drug of choice in those cases.

The clinical impact of in-hospital cerebral cardiopulmonary resuscitation maneuvers was very important in our sample. The number of patients requiring treatment to save 1 life in 12 months was 4 in our sample, similar to that found in the most recent prospective studies ${ }^{9,13,16,20}$. Few therapies in medicine have an impact on diminishing mortality like cerebral cardiopulmonary resuscitation does (tab. V). We believe that, as in out-of-hospital cardiopulmonary arrest and its chain of survival, the medical society should invest in training and establishing programs that aim at improving the assistance of patients with in-hospital cardiopulmonary arrest.

Training nurses in emergency cardiac care according to the guidelines of the AHA caused an increase in survival after in-hospital cardiopulmonary arrest from 10 to $38 \%$

\begin{tabular}{|c|c|c|}
\hline \multicolumn{3}{|c|}{$\begin{array}{c}\text { Table V - Table of impact of the different therapies in cardiovascular } \\
\text { medicine }\end{array}$} \\
\hline Intervention & Outcomes in years & NNT \\
\hline Enalapril in HF class IV & 1 death & 3 \\
\hline In-hospital CCPR & 1 death (1 year) & 3.6 \\
\hline MRC in lesion of TCE & 1 death & 6 \\
\hline Treatment of severe SAH & 1 death/AMI/AVE & 8 \\
\hline Carotid endarterectomy & 1 death/AVE & 9 \\
\hline ASA in unstable angina & 1 death/AMI & 12 \\
\hline Sinvastatin in DAS & 1 coronary death & 12 \\
\hline Enalapril in HF class II/III & 1 death & 50 \\
\hline ASA in healthy physicians & 1 death/AMI & 250 \\
\hline Treatment of mild SAH & 1 death/AMI/AVE & 350 \\
\hline \multicolumn{3}{|c|}{$\begin{array}{l}\text { NNT- necessary number of patients treated; HF- heart failure; CCPR- cere- } \\
\text { bral cardiopulmonary resuscitation; MRC- myocardial revascularization } \\
\text { surgery; SAH- systemic arterial hypertension; ASA- acetylsalicylic acid; } \\
\text { CAD- coronary artery disease. Source: Braunwald et al, Heart Disease, 2000, } \\
6^{\text {th }} \text { ed. * NNT in our sample was calculated to save } 1 \text { life in } 12 \text { months. }\end{array}$} \\
\hline
\end{tabular}

$(\mathrm{p}<0.0 .1)$ in a study carried out by Dane et $\mathrm{al}^{13}$. McGowan et al ${ }^{20}$ trained nurse's aides, nurses and physicians at their hospital and significantly improved the survival rates; Henderson et $\mathrm{al}^{21}$ reported an increase in the resuscitation rates from 30 to $58 \%(\mathrm{p}<0.0002)$ in their hospital with the creation of a special team to assist cardiopulmonary arrests. At the Hospital São Lucas, a training program on cerebral cardiopulmonary resuscitation was already ongoing during data collection of our study involving nurse's aides, nurses, and medical residents at the hospital, in 600 people being trained according to the guidelines of the AHA each 6 months ${ }^{18}$.

We believe that not using the complete protocol in the Utstein style model in in-hospital cardiopulmonary arrest ${ }^{22}$ in our study may somehow have made the comparison between our results and those from studies published in the literature difficult. However, we emphasize that most variables required by that protocol were analyzed.

In our study, maintenance of a pulse for at least 1 hour was required to define the re-establishment of spontaneous circulation, which was different from the definition adopted in the Utstein Style model, in which any return of pulse is considered a re-establishment of spontaneous circulation. If on the one hand that stricter criterion may underestimate the initial success of cardiopulmonary arrest, on the other hand, it may increase the validity of the findings.

The loss of 12 cases during data collection may have impaired the results, but we believe it is a reasonable number due to technical difficulty in performing the study. We emphasize that the strategy adopted in this study is considered ideal for the Utstein Style model for in-hospital cardiopulmonary arrest ${ }^{22}$.

In conclusion, coronary artery disease, septic shock, and pulmonary thromboembolism were the most frequently found causes of cardiopulmonary arrest. Ventricular fibrillation or tachycardia as the initial rhythm, the shorter times of cardiopulmonary arrest, cerebral cardiopulmonary resuscitation, and the higher value of mean blood pressure prior to cardiopulmonary arrest were independent variables of a better prognosis for the outcomes re-establishment of spontaneous circulation and hospital discharge. The longer time of hospitalization prior to cardiopulmonary arrest was considered a variable of a better prognosis only for the outcome hospital discharge after in-hospital cerebral cardiopulmonary resuscitation. Finally, we conclude that, in our sample, in-hospital cerebral cardiopulmonary resuscitation has a great impact in diminishing mortality as compared with other cardiovascular therapies currently used in evidence-based medicine.

\section{Acknowledgments}

We thank the Fundo de Amparo a Pesquisa do Rio Grande do Sul (FAPERGS) and the Conselho Nacional de Pesquisa (CNPQ). We also thank Drs. Ivana Beatrice Mânica Cruz and Manuela Cavalcanti for their support in the statistical analysis, and Drs. Carlos Kalil and Euler Manenti for their help with data collection. 


\section{References}

1. Kouwhenhoven WB, Jude JJ. Closed-chest cardiac massage. JAMA 1960; 37; 10: $94-7$.

2. Hillman K, Parr M, Stewart A. Redefining in-hospital resuscitation: the concept of the medical emergency team. Resuscitation 2001; 48: 105-10.

3. Eisenberg MS, Mengert TJ. Primary care: cardiac resuscitation. N Eng J Med 2001; 344: 17

4. Ballew KA, Philbrick JT. Causes of variation in reported in-hospital CPR survival: a critical review. Resuscitation 1995; 30: 203-15.

5. Eisenberg MS, Bergner L, Hallstrom A. Cardiac resuscitation in the community: importance of rapid provision and implications for program planning. JAMA 1979; 241: 1905-7.

6. Larsen MP, Eisenberg MS, Cummins RO, Hallstrom AP. Predicting survival from out-of-hospital cardiac arrest: a graphic model. Ann Emerg Med 1993; 22: 1652-8.

7. Copper S, Code J. Predicting survival, in-hospital cardiac arrest: resuscitation survival variables and training effectiveness. Resuscitation 1997; 33: 17-21.

8. Cummins RO, Chamberlain DA, Abramnson NS, et al. Recommended guidelines for uniform reporting of data from out-of-hospital cardiac arrest: the Utstein style. A statement for health professionals from a task force of the American Heart Association, the European Association Council, the Heart and Stroke Foundation of Canada and the Australian Resuscitation Council. Circulation 1991; 84: 960-75.

9. Andreásson A, Herlitz J, Bâng A, et al. Characteristics and outcomes among patients with a suspect in-hospital cardiac arrest. Resuscitation 1998; 39: 23-31.

10. Gwinnutt C, Clumb M, Harris R. Outcome after cardiac arrest in adults in UK hospitals: effect of the 1997 guidelines. Resuscitation 2000; 47: 125-35.

11. Böttiger BW, Bode C, Martin E, et al. Efficacy and safety of thrombolytic therapy after initially unsuccessful cardiopulmonary resuscitation: a prospective clinical trial. Lancet 2001; 357: 1583-5.
12. Timerman A, Sauaia N, Piegas L, et al. Prognostic factors of the results of cardiopulmonary resuscitation in a cardiology hospital. Arq Bras Cardiol 2001; 77.

13. Dane F, Katherine S, Brown TD. In-hospital resuscitation: association between ACLS training and survival to discharge. Resuscitation 2000; 47: 83-7.

14. Ballew AK, Philbrick JT. Causes of variation in reported in-hospital CPR survival: a critical review. Resuscitation 1995; 30: 203-15.

15. Herlitz J, Andreásson A, Lindqvist J, et al. Long-term prognosis among survivors after in-hospital cardiac arrest. Resuscitation 2000; 45: 167-77.

16. Cummins RO, Eisenberg MS, Sults KR. Automatic external defibrillators: clinical issues for cardiology. Circulation 1986; 73: 381-5.

17. Spearpoint $K$, Mclean $P, Z$ Zideman D. Early defibrillation and the chain of survival inhospital adult cardiac arrest; minutes count. Resuscitation 2000; 44: 1655-169.

18. Kudenchuk P, Cobb L,Walsh T, et al. Amiodarone for resuscitation after out-ofhospital cardiac arrest due to ventricular fibrillation. N Engl J Med 1999; 341: 871-8.

19. Guidelines 2000 for Cardiopulmonary Resuscitation and Emergency Cardiovascular Care. An International Consensus on Science. The American Heart Association in Collaboration With the International Liaison committee on Resuscitation. Circulation 2000; 102(suppl I): I-1-I-11.

20. McGowan J, Colin G, Gordon M. Appointment of a Resuscitation Training Officer is associated with improved survival from in-hospital ventricular fibrillation/ventricular tachycardia cardiac arrest. Resuscitation 1999; 41: 169-73.

21. Henderson S, Ballestros D. Evaluation of a hospital-wide resuscitation team: does it increase survival for in-hospital cardiopulmonary arrest? Resuscitation 2001; 48: 111-6.

22. Cummins R, Chamberlein D, Cobbe Stuart, et al. Recommended guidelines for reviewing, reporting, and conducting research on in-hospital resuscitation: the in-hospital "Utstein style". Resuscitation 1997; 34: 151-83. 